\title{
Revista Colombiana de

\section{Mecánica rotacional ventricular izquierda invertida en un paciente con lipedema (del estudio de MAGYAR-Path)}

\author{
Attila Nemes ${ }^{a}$, Árpád Kormányos ${ }^{a}$, Péter Domsik ${ }^{a}$, Isabel Forner-Cordero ${ }^{b}$, \\ Lajos Kemény ${ }^{c}$ y Győző Szolnoky ${ }^{c, *}$
}

\author{
a 2nd. Department of Medicine and Cardiology Centre, Faculty of Medicine, Albert Szent-Györgyi Clinical Center, University of \\ Szeged, Szeged, Hungría \\ ${ }^{\mathrm{b}}$ Lymphedema Unit. Hospital Universitari i Politècnic La Fe, University of Valencia, Valencia, España \\ c Department of Dermatology and Allergology, Faculty of Medicine, Albert Szent-Györgyi Clinical Center, University of Szeged, \\ Szeged, Hungría
}

Recibido el 3 de enero de 2018; aceptado el 1 de mayo de 2018

Disponible en Internet el 2 de octubre de 2018

\section{PALABRAS CLAVE \\ Lipedema; \\ 3D ecocardiografia; \\ Rotación; \\ Lipedema}

\section{KEYWORDS}

Lipoedema;

3-D echocardiograph;

Rotation;

Lipoedema

\begin{abstract}
Resumen El lipedema es un trastorno crónico que se caracteriza por una hiperplasia del tejido adiposo subcutáneo simétrico, deformante, asociado a hematomas y dolor, que afecta fundamentalmente a mujeres tras la pubertad, que suele ser familiar y tiene influencia hormonal. Sin tratamiento, el lipedema puede progresar a linfedema, y se ha asociado al aumento en la rigidez de la aorta. La mecánica rotacional del ventrículo izquierdo (VI) tiene un papel significativo en la circulación normal. En circunstancias normales, la base del VI rota en el sentido de las agujas del reloj, mientras que el ápex del VI rota en sentido contrario, provocando un movimiento como de retorcer una toalla llamado torsión del VI. En el presente caso, mediante ecocardiografía speckle-tracking tridimensional se reveló una torsión del VI demostrando un ápex $\mathrm{VI}$ en el sentido de las agujas del reloj y la rotación antihoraria de la base del VI en un paciente con características clínicas de lipedema.

( ) 2018 Sociedad Colombiana de Cardiología y Cirugía Cardiovascular. Publicado por Elsevier España, S.L.U. Este es un artículo Open Access bajo la licencia CC BY-NC-ND (http:// creativecommons.org/licenses/by-nc-nd/4.0/).
\end{abstract}

Left ventricular twist rotation mechanics in a patient with lipoedema (MAGYAR-Path Study)

Abstract Lipoedema is a chronic disorder that is characterised by a symmetric, deforming, hyperplasia of the subcutaneous adipose tissue, and is associated with haematomas and pain. It mainly affects women after puberty, is usually familial and is influence by hormones. Untreated,

\footnotetext{
* Autor para correspondencia.

Correo electrónico: szolnokygyozo@gmail.com (G. Szolnoky).
} 
the lipoedema can progress to lymphoedema, and has been associated with the increase in aortic stiffness. The mechanical rotation of the left ventricle (LV) has an important role in normal circulation. In normal circumstances, the base of the LV rotates clockwise, while the LV apex rotates in the opposite direction, leading to a movement like wringing a towel, called LV twist. In the present case, using three-dimension speckle-tracking echocardiography, an LV twist is observed, showing an LV apex in the clockwise direction and the anti-clockwise rotation of the LV base in a patient with clinical characteristics of lipoedema.

(c) 2018 Sociedad Colombiana de Cardiología y Cirugía Cardiovascular. Published by Elsevier España, S.L.U. This is an open access article under the CC BY-NC-ND license (http:// creativecommons.org/licenses/by-nc-nd/4.0/).

El lipedema es un trastorno crónico caracterizado por hiperplasia del tejido adiposo asociada a hematomas espontáneos o ante mínimos traumatismos y dolor neuropático, que afecta fundamentalmente a mujeres tras la pubertad ${ }^{1}$. El diagnóstico clínico es relativamente fácil; sin embargo, los conocimientos respecto a sus mecanismos fisiopatológicos son más que limitados. El diagnóstico diferencial se hace con linfedema, obesidad, lipohipertrofia y flebedema. El lipedema se presenta con un aumento simétrico y desproporcionado de tejido adiposo desde caderas y muslos y además se caracteriza por dolor y hematomas espontáneos o inducidos por traumatismos menores. La enfermedad no afecta a los pies ni el área baja del tobillo. Se distinguen cinco tipos:

Tipo I: el desarrollo de tejido graso lipedematoso afecta a glúteos y muslos.

Tipo II: el lipedema se extiende a las rodillas con formación de almohadillas grasas en la cara interna de las rodillas.

Tipo III: el lipedema se extiende de las caderas a los tobillos.

Tipo IV: los brazos y las piernas son lipedematosos.

Tipo V: el lipedema afecta a las piernas.

Según la gravedad de los síntomas clínicos, el lipedema se clasifica en tres estadios: I: piel de apariencia normal y subcutis de consistencia blanda aunque se pueden palpar múltiples nódulos; II: superficie cutánea irregular y dura a causa de su estructura nodular, con nódulos subcutaneos más grandes (lipoesclerosis); III: deformación lobular de la piel debido al aumento de tejido adiposo con nódulos de tamaños variables ${ }^{1}$.

Los protocolos terapéuticos comprenden el tratamiento conservador (terapia descongestiva de linfedema) y quirúrgico (liposucción). A largo plazo, la liposucción aporta beneficios notables y duraderos, en términos de reducción de volumen y de síntomas subjetivos. El diagnóstico y tratamiento precoces son primordiales, ya que el aumento gradual del depósito de grasa causa pérdida de movilidad y otras comorbilidades como artrosis y linfedema ${ }^{1}$.

En circunstancias normales, la base del ventrículo izquierdo (VI) rota en el sentido de las agujas del reloj, mientras que el ápex del VI lo hace en sentido contrario, cuusando un movimiento conocido como torsión ventricular del $\mathrm{VI}^{2}$. La mecánica rotacional del VI tiene un papel significativo en la circulación normal y aproximadamente el $40 \%$ de la eyección se debe a la mecánica de rotación del VI según los conocimientos científicos recientes ${ }^{3}$.

Recientemente, el aumento en la rigidez de la aorta se ha asociado con el lipedema ${ }^{4}$; por tanto, debido al acoplamiento arterial-ventricular, las alteraciones en la rotación del VI y el giro podrían ser esperables en el lipedema ${ }^{3}$.

Se expone el caso de una mujer no fumadora, normotensa, de 60 años de edad, con características clínicas de lipedema estadio 2, quien se reclutó en el Motion Analysis of the heart and Great vessels bY three-dimensionAl speckle-tRacking echocardiography in Pathological cases (MAGYAR-Path) Study. Tenía antecedentes de un episodio único, en 2010, de trombosis venosa profunda en la pierna izquierda y tromboembolia pulmonar, por lo que seguía en tratamiento con dabigatrán oral (110 mg b.i.d.).

Se empleó un equipo de ecocardiografía disponible comercialmente Toshiba Artida ${ }^{\mathrm{TM}}$ (Toshiba Medical Systems, Tokyo, Japan), para la ecodoppler bidimensional (2D) y la ecocardiografía speckle tracking (rastreo de marcas) tridimensional (3DSTE). Se utilizó un transductor matricial de 1-5 MHz PST-30SBP para la ecocardiografía en 2D y un transductor matrix matricial de $1-4 \mathrm{MHz}$ PST-25SX para la adquisición en 3D durante la 3DSTE. Para las cuantificaciones de las cámaras, se empleó el software Artida 3D Wall Motion Tracking, version 2.7. Las imágenes dos y cuatro cámaras apicales se usaron para marcar manualmente los bordes del endocardio del VI a nivel de la válvula mitral a cada lado, así como a nivel del ápex del VI, y luego el software realizó una reconstrucción automática de la superficie endocárdica en $3 D$ y lo monitorizó en 3D durante el ciclo cardiaco (fig. 1A). Del molde reconstruido en $3 \mathrm{D}$ del $\mathrm{VI}$, se calcularon las rotaciones apical, medioventricular y basal; al igual que el giro y los valores de strain total, radial, longitudinal y circunferencial.

Para el análisis de los datos y la creación de gráficos se empleó un paquete de software comercial (MATLAB 8.6, The MathWorks Inc., Natick, MA, 2015).

Durante la ecocardiografía en 2D, se observaron las dimensiones normales de las cámaras y parámetros funcionales normales. Los valores de 3DSTE-derived strain total, radial, longitudinal, circunferencial y $3 \mathrm{D}$ fueron $30,0 \%$, $-15,4 \%, 22,2 \% ;-34,5 \%$ y 32,3\%, respectivamente. Se observó que la rotación apical del VI era en dirección a las agujas del reloj, mientras que la base del VI tenía una rotación 

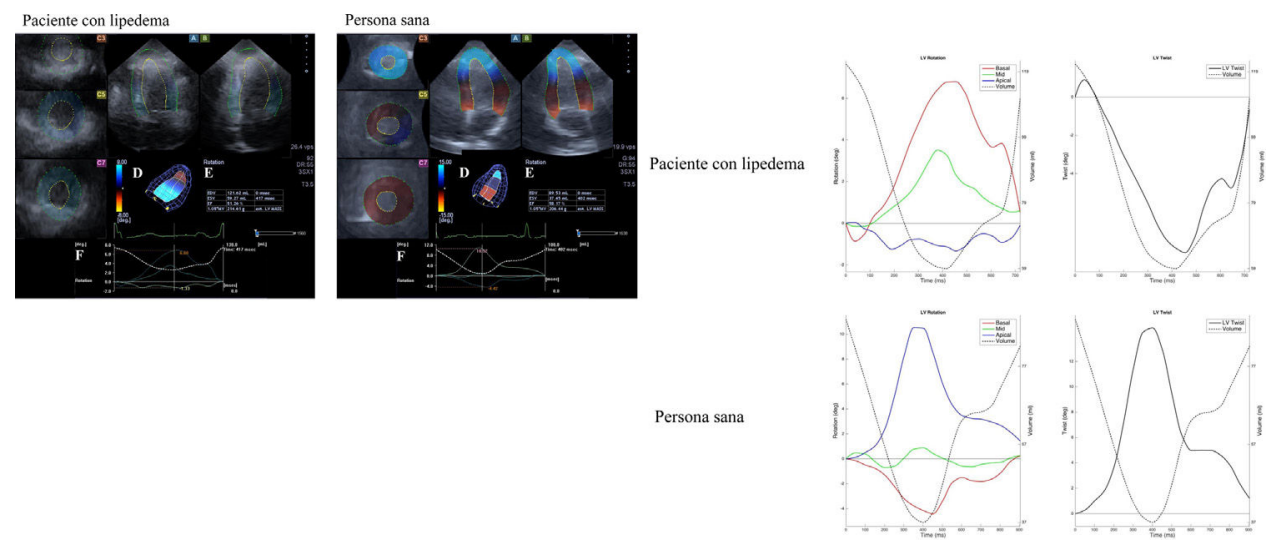

Figura 1 A Imágenes apicales de cuatro- (A) y dos cámaras (B) y tres vistas de eje corto $(C 3, C 5, C 7)$, molde tridimensional del ventrículo izquierdo (VI) (D) y datos volumétricos (E) del paciente lipedemático y el de un sujeto femenino control se presentan junto al tiempo - curvas rotacionales apicales del VI, medioventriculares y basales (F). La curva discontinua representa el cambio de volumen del VI durante el ciclo cardiaco. B Reconstrucción de las curvas rotacionales del ventrículo izquierdo (VI) del paciente con lipedema y de un sujeto sano apareado. En circunstancias normales, se observaría una rotación apical del VI en sentido antihorario y una rotación basal del VI en sentido horario. En el presente caso de lipedema, se demostraron rotaciones apical y basal invertidas.

antihoraria, sugiriendo una torsión invertida del VI en este caso (fig. 1B). Además, ambas rotaciones, apical y basal del VI, mostraron estar disminuidas en su extensión resultando en una torsión no sólo invertida sino reducida del VI.

Hasta donde los autores conocen, esta es la primera vez que se examina y se presenta la mecánica rotacional del VI en un paciente con lipedema. La base del VI rotó en sentido antihorario, mientras la rotación apical ocurrió en el sentido de las agujas del reloj, sugiriendo una inversión de la mecánica rotacional del VI en este caso. Además, las rotaciones apical y basal del VI y, por tanto, el giro del VI, mostraron estar significativamente disminuidos, lo cual sugiere que no sólo su dirección sino su extensión, también están alteradas.

En circunstancias normales, las fibras miocárdicas de las capas subendocárdicas y subepicárdicas del VI son responsables del giro del VI debido a su disposición en direcciones opuestas $^{3,5}$. Las fibras miocárdicas se disponen en sentido izquierdo en el subepicardio y en sentido derecho en el subendocardio. La dirección del giro del VI en el pico sistólico es gobernada por las fibras subepicárdicas, debido sobre todo a su gran brazo de movimiento ${ }^{3,5}$. En consecuencia, en algunos estados patológicos que afectan al subendocardio, como la isquemia o la miocardiopatía hipertrófica, se puede detectar una hiperrotación del $\mathrm{VI}$ y un aumento del giro del $\mathrm{VI}^{3}$. Además, tanto las miocardiopatías como las enfermedades cardiacas infiltrativas y congénitas se asocian frecuentemente con la casi ausencia del giro del VI, llamado rigid body rotation ${ }^{5,6}$. Sin embargo, en estos trastornos no se ha encontrado una inversión en la mecánica rotacional del VI.

En teoría, la rigidez elevada de la aorta ${ }^{7}$, la anatomía y la función del corazón en pacientes con lipedema que es semejante a la miocardiopatía dilatada ${ }^{8}$ (datos no mostrados), la posible alteración en la composición del miocardio ${ }^{9}$ (el depósito de lípidos, fibrosis local, etc), los efectos de la trombosis venosa profunda y la embolización pulmonar a través de la sobrecarga de ventrículo derecho con efecto sobre el VI (alteración en la presión transmural y precarga -preload- reducida) juntos podrían conducir a las anomalías rotacionales del VI.
Además, no se pueden descartar razones hemodinámicas relacionadas con el lipedema. Se precisan nuevos estudios que examinen la mecánica rotacional del VI en una serie de pacientes con características clínicas típicas de lipedema. Igualmente, sería conveniente investigar el origen fisiopatogénico de la inversión de la torsión del VI y examinar su prevalencia e importancia clínica en el lipedema y en otros grupos de pacientes.

\section{Financiación}

Bauerfeind Premio de Flebología 2015-2017 (UnidadInternacional de Flebología).

\section{Normas éticas}

Los autores afirman que todos los procedimientos que contribuyen a este trabajo cumplen con las normas éticas de las guías más relevantes nacionales sobre experimentación humana y con la Declaración de Helsinki de 1975, revisada en 2008, y ha sido aprobado por el Comité institucional de la Universidad de Szeged (Hungría).

\section{Conflicto de intereses}

Ninguno.

\section{Bibliografía}

1. Forner-Cordero I, Szolnoky G, Forner-Cordero A, Kemény L. Lipedema: an overview of its clinical manifestations, diagnosis and treatment of the disproportional fatty deposition syndrome - systematic review. Clin Obes. 2012;2:85-96.

2. Faconti L, Bruno RM, Buralli S, Barzacchi M, Dal Canto E, Ghiadoni L, Taddei S. Arterial-ventricular coupling and parameters of vascular stiffness in hypertensive patients: Role of gender. JRSM Cardiovasc Dis. 2017;6, 2048004017692277. 
3. Nakatani S. Left ventricular rotation and twist: why should we learn? J Cardiovasc Ultrasound. 2011;19:1-6.

4. Szolnoky G, Nemes A, Gavallér H, Forster T, Kemény L. Lipedema is associated with increased aortic stiffness. Lymphology. 2012;45:71-9.

5. van Dalen BM, Caliskan K, Soliman OI, Nemes A, Vletter WB, Ten Cate FJ, Geleijnse ML. Left ventricular solid body rotation in non-compaction cardiomyopathy: a potential new objective and quantitative functional diagnostic criterion? Eur J Heart Fail. 2008;10:1088-93.

6. Nemes A, Földeák D, Domsik P, Kalapos A, Sepp R, Borbényi Z, Forster T. Different patterns of left ventricular rotational mechanics in cardiac amyloidosis-results from the three-dimensional speckle-tracking echocardiographic MAGYAR-Path Study. Quant Imaging Med Surg. 2015;5:853-7.

7. Gnakamene JB, Safar ME, Levy BI, Escoubet B. Left ventricular torsion associated with aortic stiffness in hypertension. J Am Heart Assoc. 2018;7.

8. Sengupta PP, Tajik AJ, Chandrasekaran K, Khandheria BK. Twist mechanics of the left ventricle: principles and application. JACC: Cardiovascular Imaging. 2008;1:366-76.

9. Varga I, Kyselovič J, Galfiova P, Danisovic L. the noncardiomyocyte cells of the heart. their possible roles in exercise-induced cardiac regeneration and remodeling. Adv Exp Med Biol. 2017;999:117-36. 